\title{
Collapse of conical granular columns
}

\author{
M.A. Ordaz ${ }^{1, *}, M$. Sánchez-Rosas $^{2}, J . R$. Hernández-Juárez ${ }^{1}, A$. Medina $^{1}$, and D.A. Serrano ${ }^{2, * *}$ \\ ${ }^{1}$ ESIME Azcapotzalco, Instituto Politécnico Nacional, Av. de las Granjas 682, Col. Sta. Catarina, Azcapotzalco 02250 CDMX. \\ ${ }^{2}$ Instituto Tecnológico de Querétaro, Av. Tecnológico s/n, Centro, 76000 Santiago de Querétaro, Qro.
}

\begin{abstract}
This paper experimentally analyzes the collapse of conical granular columns made out of non-cohesive granular materials. Dimensionless run-out lengths were found, as a function of the aspect ratio $a$, similar to those occurring in the collapse of cylindrical columns, although in the case of conical columns of high aspect ration, at the end of the collapse, a layer of grains that rotates around the cone axis appears and it affects the final profiles of the mounds.
\end{abstract}

\section{Introduction}

The collapse of granular columns typically is characterized through the sudden spreading, due to gravity, of dense, non-cohesive granular material until the flow of grains is halt. Such a scenario has interesting analogies with natural accumulations of granular material, of different shapes, which sometimes tend to move suddenly, due to gravity. For instance, the occurrence of landslides due to heavy rainfalls (mud or dry soil) is very common each year and consequently many people are exposed to this type of disasters.

In a laboratory, cylindrical granular columns are allowed to relax to stable configurations by slumping on horizontal surfaces. It was observed that the normalized runout lengths of axisymmetric mounds obey different power-laws, depending on the initial aspect ratio of the columns, $a$.

Here we are interested in the experimental investigation of the collapse of conical, densely packed, granular columns in order to explore if there are substantial differences with respect to collapse in cylindrical granular columns due to the inherent existence of an overall slight slope and a nonuniform weight distribution. As one of the main results of our experiments, we report that, for large aspect ratios, at the last stage of the slumping, a skew of the grains occurs and the final shape of the mound is non axisymmetric, at the top. However, we also found that the normalized runout lengths, for the conical columns, obey power-laws similar to those occurring in cylindrical columns. In order to reach our goal, this work is divided as follows: in Section 2 we briefly review the main methods of characterization of the columns before and after their collapses. In Section 3 we present experimental results

\footnotetext{
*e-mail: amedinao@ipn.mx

**e-mail: arman2390@ @otmail.com

A video is available at https://doi.org/10.48448/ah9r-v551
}

for conical and cylindrical columns, and finally in Section 4 we give the main conclusions of this work.

\section{Previous work in cylindrical columns}

The investigation of the slumping of axisymmetric and non axisymmetric granular columns, has been carried out experimentally [1,2] and numerically [3]. This kind of experiments have been conducted not only in granular columns, but also with water columns [4, 5]. Several studies also directed their research to the long rectangular geometries varying the width and height of the columns in water [6].

Through laboratory experiments Lube et al [1] determined the normalized runout lengths due to the collapse of cylindrical granular columns of initial radius $R_{0}$ and height $H_{0}$, respectively. They used different aspect ratios, $a=H_{0} / R_{0}$ and different materials such as salt, sugar, rice and sand. After the collapse onto a flat plane, the final deposit is characterized by the runout distance $R_{\infty}$ and the height $H_{\infty}$. The leading results for the dimensionless runout length $\left(R_{\infty}-R_{0}\right) / R_{0}$, as a function of $a$, obey the relationships

$$
\frac{R_{\infty}-R_{0}}{R_{0}} \cong\left\{\begin{array}{r}
1.24 a, a \leq 1.7 \\
1.60 a^{1 / 2}, a \geq 1.7
\end{array}\right.
$$

Lajeunesse et al [2], for a half-cylindrical geometry, used four different substrate materials with the same start up conditions, experimentally obtained the scaled runout length

$$
\frac{R_{\infty}-R_{0}}{R_{0}} \simeq\left\{\begin{aligned}
1.35 a, a & \lesssim 0.74 \\
2.0 a^{1 / 2}, a & \gtrsim 0.74 .
\end{aligned}\right.
$$

Later, Staron and Hinch [3], using the discrete element method, performed several numerical experiments for different values of the aspect ratio, obtaining very similar results to those by Lube et al [1], finding 


$$
\frac{R_{\infty}-R_{0}}{R_{0}} \cong\left\{\begin{array}{r}
2.5 a, a \lesssim 2 \\
3.25 a^{\alpha}, a \gtrsim 2,
\end{array}\right.
$$

where $\alpha=0.705 \pm 0.022$.

Now, we report experiments performed with conical granular columns because, in nature and industry, it is common to find conical-like mounds. Experiments of slumping with cylindrical columns were also carried out to compare with results for conical columns.

\section{Experiments with conical and cylindrical columns}

In our experiments, collapse of conical columns was performed by partially filling glass-made cones with non-cohesive materials like beach sand (angulated sand grains), Ottawa sand (rounded sand grains) and granulated sugar (near crystalline grains). Properties of these materials are given in Table 1 . Similarly, the main characteristics of the frustum-like columns are given in Table 2; it should be mentioned that we will make the comparison with cylindrical columns of radius equal to the lower radius of the conical columns.

The tests of the granular collapses of conical columns, were carried out on a flat, paper sheet with a printed length scale. In Fig. 1 we show a schematic of the cone and the main dimensions of the initial granular column and a specific final profile.

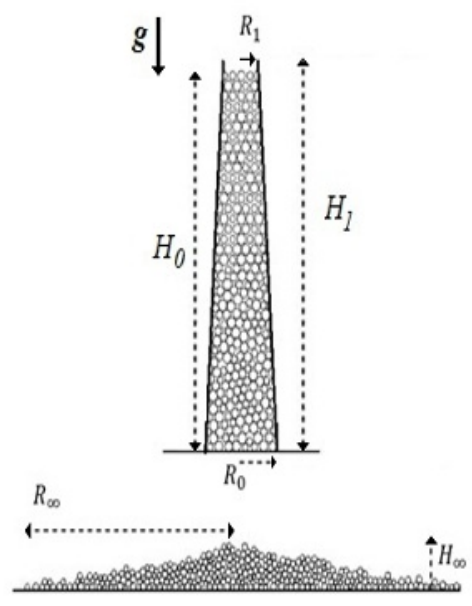

Figure 1. Schematic of a glass-made cone partially filled with a granular material, the dimensions of the cone are given in the upper drawing. When the glass cone is suddenly removed, the released conical column slumps onto a flat bottom plane and the new dimensions of the resulting cone are given in the lower drawing.

In cylindrical columns, the aspect ration $a$ is given as the ratio of the initial height of the column $H_{0}$ and the internal radius $R_{0}$, meanwhile in conical columns we define the aspect ratio as $a=H_{0} / R_{m}$ where the average radius is $R_{m}=\left(R_{0}+R_{1}\right) / 2$ and $R_{1}$ is the corresponding radius of the granular column at the top. For all materials tested we performed experiments with all the aspect ratios of Table 2 .

\begin{tabular}{|l|l|l|l|}
\hline Material & $\begin{array}{l}\text { Bulk } \\
\text { density }\end{array}$ & $\begin{array}{l}\text { Mean grain size } \\
\text { (median of the } \\
\text { size distribution) } \\
\mathrm{mm}\end{array}$ & $\begin{array}{l}\text { Friction } \\
\text { angle }\end{array}$ \\
$\mathrm{gr} / \mathrm{cm}^{3}$ & Degs \\
\hline Beach sand & 2.6 & 0.30 & 30 \\
\hline Ottawa sand & 2.51 & 0.85 & 36 \\
\hline Granulated sugar & 1.58 & 0.73 & 35 \\
\hline
\end{tabular}

Table 1. Properties of the particles used in the experiment.

The container filling process was carried out carefully and systematically by gently pouring the grains from the upper aperture of the cone (or cylinder) upto a predetermined height. Verticality of the axis of the cone was checked, before each experiment, using a plumb-line. In each experiment, the cone was rapidly raised at speeds of about $2 \mathrm{~m} / \mathrm{s}$, then, the column slumps due to the gravity action, the overall grains motion last typically less than one second, as seen in the snapshots in Fig. 2.

The runout distance $R_{\infty}$ and height of the final deposit $H_{\infty}$ were also measured. To observe the dynamics of the column collapse the events were video recorded at a rate of $250 \mathrm{fps}$, it allowed us to have very definite profiles of the evolving granular column. By using a software for mechanical design and the scale printed on the surface we were able to measure have had the capability to measure vertical and horizontal motion of grains at any point of the slumping column, but here we mainly report the runout distances at the verge.

At the last instants of the collapse the granular column developed a spinning with respect to its vertical axis, if $a$ is large, for all materials. We argument that a rotational force arises because in conical columns there appears a non-uniform torque $\tau$, given by the cross product of the form $\tau=\mathbf{r} \times \mathbf{F}$, where the vector $\mathbf{r}$ coincides with the polar-coordinate and $\mathbf{F}$ is the tangential, downward force vector, due to the non-uniform weight and frictional force.

\begin{tabular}{|l|l|l|}
\hline $\begin{array}{l}R_{0} \\
\mathrm{~cm}\end{array}$ & $\begin{array}{l}R_{1} \\
\mathrm{~cm}\end{array}$ & $\begin{array}{l}\text { Aspect Ratio } \\
a=H_{0} / R_{m}\end{array}$ \\
\hline 2.85 & $\begin{array}{l}2.8,2.7,2.65,2.45 \\
2.3,2.1,1.95\end{array}$ & $0.35,1.08,1.81,3.77$, \\
& $5.8,8.08,10.41$ \\
\hline
\end{tabular}

Table 2. Internal lower and upper radius of the granular columns and the respective aspect ratios used in experiments.

After the collapse, at the equilibrium state, the runout distances $R_{\infty}$ and the final maximum height of the deposit $H_{\infty}$ were measured. In Fig. 3 we show the plot of dimensionless runout distance $v s$ the aspect radio $a$ for all the conical columns and materials here employed. 


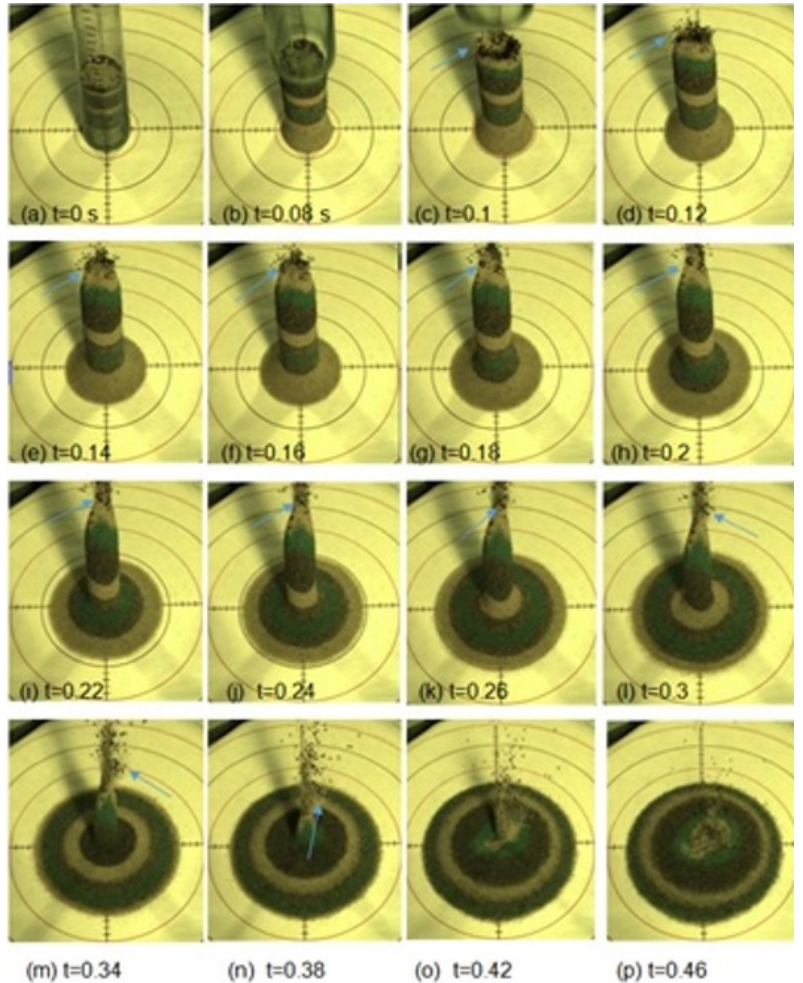

Figure 2. Snapshots of the collapse of a beach sand conical column. Initially, the granular material evolves on the horizontal surface axi-symmetrically (colored layers) but at the end there are ellipse-like deformations at the top. Mustard seeds were added to the top of the column to make its spin more evident.

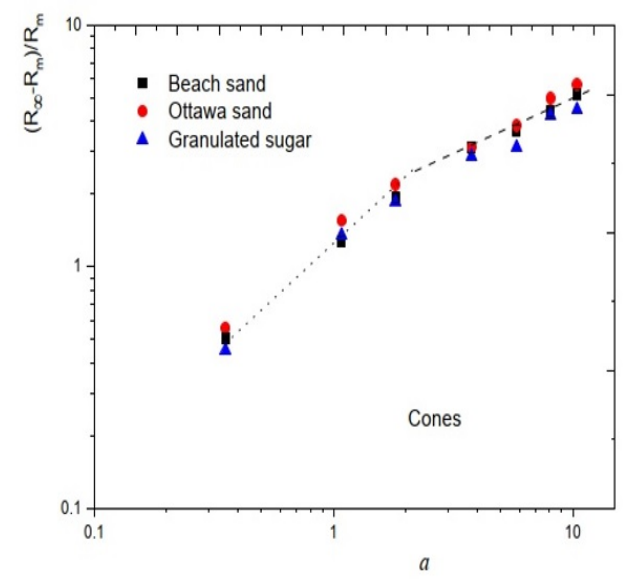

Figure 3. Log-log plot of the dimensionless runout distance versus $a$ for conical columns of several types of materials. Formula (4) fits, on average, data (dotted and dashed lines).

The relationship (4) gives the average of the best fits for each material of the dimensionless runout $v s a$ and, similar to the fits in the collapse of cylindrical columns, this fit obeys the piecewise formula

$$
\frac{R_{\infty}-R_{m}}{R_{m}} \cong\left\{\begin{array}{l}
1.20 a^{0.90 \pm 0.05}, a \lesssim 2 \\
1.47 a^{0.52 \pm 0.05}, a \gtrsim 2
\end{array}\right.
$$

Such a fit gives values of the exponent very close to those reported for cylindrical columns. Actually, in (4) the power-law terms are robust, meanwhile the prefactors change slightly for each material, because the prefactor "is related to the mass ejection from the initial column itself" [3], which is strongly related to the elastic and frictional contact among grains [7].

In order to compare with the collapse of cylindrical columns, in Fig. 4 we show the dimensionless runout distance against $a$, the average of the best fits for each material yields

$$
\frac{R_{\infty}-R_{0}}{R_{0}} \cong\left\{\begin{array}{l}
1.45 a^{0.85 \pm 0.05}, a \lesssim 2 \\
1.97 a^{0.47 \pm 0.05}, a \gtrsim 2,
\end{array}\right.
$$

again, we argued that the power laws are robust and the slight deviation respect to the straight lines may be due to the different collisional and frictional properties among grains.

In Fig. 5 it is possible to visualize the top of the conical column during the collapse, we found that a triangular concave orifice (crater) appears at this top. The experiment was performed in a column of aspect ratio $a=5.82$ by coloring the upper $5 \mathrm{~cm}$ of the total height. This kind of crater occurs just after the glass cone is completely removed, and its initiation due to disturbance caused by lifting the cone cannot be excluded. In experiments of the collapse of cylindrical columns, the moving crater detected on the lower mound, in experiments of collapse of cylindrical columns, whose behaviour is wavelike, as a wave, apparently shares the same origin [8]. More studies along this line are necessary.

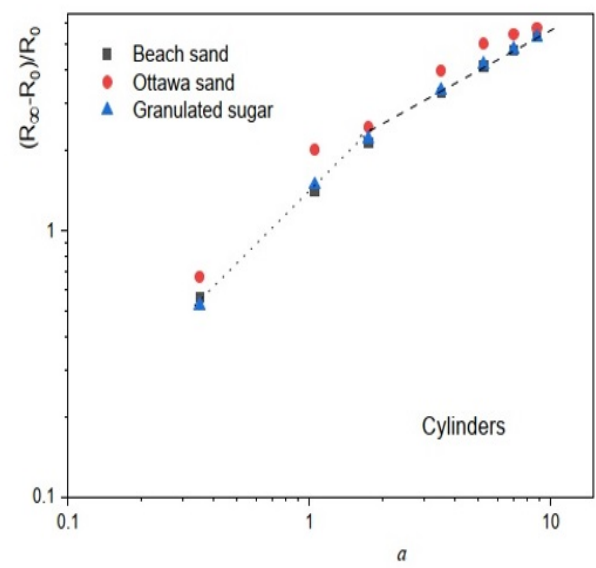

Figure 4. Log-log plot of the dimensionless runout distance versus $a$ for cylindrical columns. Formula (5) fits, on average, data (dotted and dashed lines).

In order to stress the idea that "the ability of the column to use its initial energy for spreading might depend on $a$ " [3], we estimate the initial potential energy of the initial conical column as

$$
U=\pi \rho g \int_{0}^{H_{0}} r^{2} z d z
$$




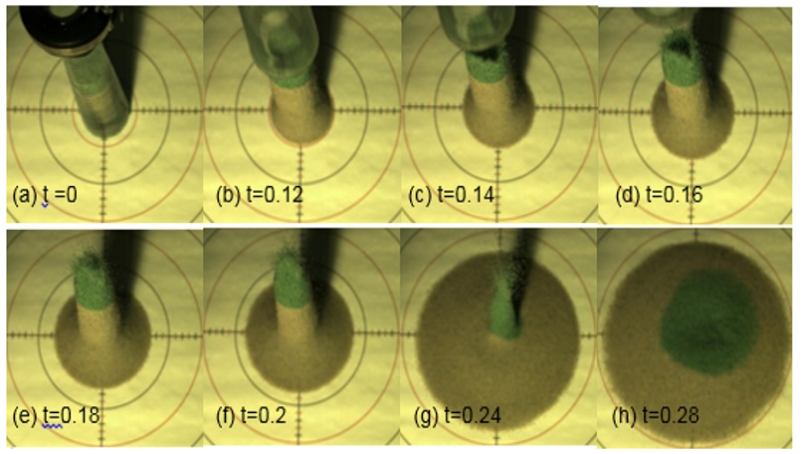

Figure 5. Snapshots of the granular collapse of a conical column performed for $a=5.82$, we colored the upper $5 \mathrm{~cm}$ of the total height. Notice the existence of a peculiar orifice (crater) at $t=$ $0.16 \mathrm{~s}$ and the asymmetry at the top of the mound caused by the skew of the grains at the upper part of the column.

where $\rho$ is the bulk density of the respective granular material and $r(z)$ is the cone profile, that in this case is $r=$ $R_{0}+m z ; z$ is the longitudinal-axis and $m=\left(R_{1}-R_{0}\right) / H_{0}$.

For the cases where $a \lesssim 1$ the final equilibrium profile is a truncated cone of the form $r=R_{\infty}+m^{*} z$, where $m^{*}=-\left(R_{\infty}-R^{*}\right) / H_{\infty}$. In such a case $H_{\infty} \simeq H_{0}$, i.e., the final height will be near $H_{0}$ and $R^{*}$ is the mound's plateau radius, to be determined through the mass conservation equations for the initial an final equilibrium cones. Then, the corresponding potential energy of this last cone is computed through the formula

$$
U=\pi \rho g \int_{0}^{H_{\infty}} r^{2} z d z .
$$

Assuming that both potential energies must be near the same, it is direct to arrive to a result of the form

$$
\left(\frac{H_{\infty}}{R_{m}}\right) \approx c a \text { for } a \lesssim 1
$$

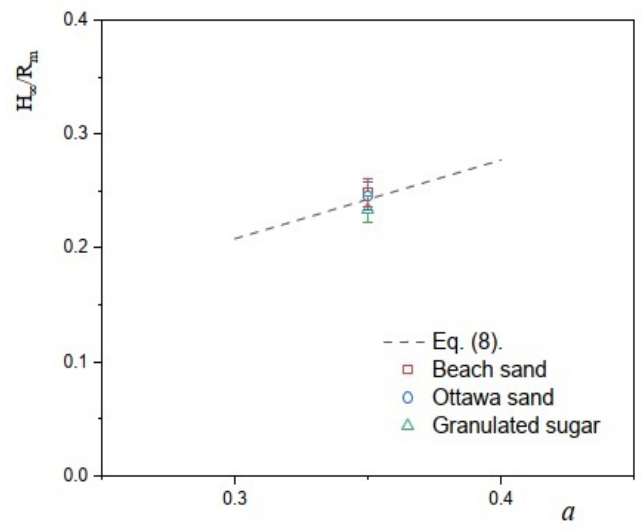

Figure 6. Plot of $H_{\infty} / R_{m}$ as a function of $a$ (Eq. (8)). Data correspond to the materials used in our experiments with $a=$ 0.35 , meanwhile the prefactor $c$ has a mean value $c=0.69$ in order to embrace the respective values of $c$ for the several materials. where $c$ is a cumbersome prefactor depending on $R_{0}, R_{1}$ and $R_{\infty}$. A power law like (8) was found by Staron et al [3] and Lajeunesse et al [2] for truncated cones derived from that for cylindrical columns (where $R_{m}=R_{0}$ ), by alluding to the scaling of the runout distance and the mass conservation. In Fig. 6 we show the plot of Eq. (8) (dashed line) and the experimental data correspond to the cases $a=$ 0.35 and the numerous materials used in this work. The fit of (8) to data confirms that the potential energy changes slightly when a frustum-like mound is obtained due to the collapse under these conditions.

\section{Conclusions}

In this report we conclude that power law formulas, as those valid for the normalized runout distances of cylindrical columns were also obtained for the runout distances in the collapse of conical columns made of beach sand, Ottawa sand and granulated sugar. Similarly, the normalized final height of the equilibrium final shapes, like frustums, obey that $H_{\infty} / R_{m} \simeq a$ for the several materials here used. Unfortunately, in plot of Fig. 6, we have a single data for each material when $a \lesssim 1$ since in our experiments our main interest was to search about the behavior of the normalized runout distances. However Eq.(8) closely fits experimental data, where the mean value of $c$ was $c=0.69$. Equation (8) was obtained by assuming the slight collapse of short columns occurs nearly without loss of potential energy, in addition to the conservation of mass. From the dynamic point of view, specially for columns where $a \gtrsim 2$, we found the formation of craters and the rotation of the upper part of the column, giving rise to a slight asymmetry in the profile of the mound. We believe that these observations will motivate more studies on the collapse in other type of geometries.

Acknowledgements.- Authors wish to thank Technological Institute of Querétaro for providing us the facilities to conduct the experiments here reported.

\section{References}

[1] G. Lube, H.E. Huppert, R.S.J. Sparks, M.A. Hallworth, J. Fluid Mech., 508, 175-199(2004).

[2] E. Lajeunesse, A. Mangeney-Castelnau, J.P. Vilotte, Phys. Fluids, 16, 2371-2381(2004).

[3] L. Staron, E.J. Hinch, J. Fluid Mech., 545, 1-27(2005).

[4] D.M. Graves, Int. J. Numer. Mech. Fluid, 50, 693-711(2006).

[5] M.A. Cruchaga, D.J. Celentano, T.E. Tezduyar, Comput. Mech., 39, 453-476(2007).

[6] A. Bougouin, L. Lacaze, Phys. Rev. Fluids, 3, 064305(2018).

[7] T. Man, H.E. Huppert, L. Li, S.A. Galindo-Torres, arXiv: 2002.02146, (2020).

[8] O. Roche, M. Attali, A. Mangeney, A. Lucas, Earth Planet. Sci. Lett. 311, 375-385(2011). 